Case Report

\title{
Fluorescence in situ hybridization on Peripheral blood for Chronic Myeloid Leukaemia - Rapid and reliable method
}

\author{
Meenakshi A. , Prashanth Shetty D. ${ }^{2}$, Suchetha Kumari N. ${ }^{3}$, Michelle Mathias ${ }^{4}$, Karuna Ramesh \\ Kumar $^{5}$ \& Jayaprakash Shetty ${ }^{6}$ \\ ${ }^{1}$ Lecturer \& Research Associate, ${ }^{2,3}$ Professors, Diagnostic Centre for Cytogenetics \& M olecular Genetics, \\ ${ }^{4,5}$ Professors, ${ }^{6}$ Professor \& HOD, Department of Pathology, K. S. Hegde M edical Academy, \\ Nitte University, Mangalore, Karnataka, India.
}

Correspondence

Meenakshi A.

Lecturer \& Research Associate, Diagnostic Centre for Cytogenetics \& M olecular Genetics, K. S. Hegde M edical Academy, Nitte University, Mangalore 575 018, Karnataka, India. Mobile : +91 7829418612 E-mail : hellomeenaa@gmail.com

\begin{abstract}
Philadelphia chromosome (Ph) is found in more than $95 \%$ of Chronic Myeloid Leukaemia $(\mathrm{CML})$ patients arising from the reciprocal translocation of chromosomes 9 and 22 which results in the formation of chimeric fusion gene BCR-ABL. This paved the path for targeted gene therapy in CML and thus plays a pivotal role in diagnosis and prognosis. Fluorescence in situ hybridization (FISH) is a rapid and reliable technique in molecular cytogenetics to detect BCR-ABLfusion signal in both interphase and metaphase spreads of bone marrow sample. Peripheral blood white cellsas a surrogate for bone marrow have been suggested by a few studies.

The objective of the study was to evaluate FISH on peripheral blood specimen as a rapid and reliable method to quantify Ph positive cells in a patient with Chronic M yeloid Leukaemia. FISH was performed on interphase nuclei from cultured peripheral blood sample of the patient using BCR/ABL Translocation, Dual fusion probe. Chromosomal analysis was performed by GTG banding technique. FISH and karyotyping confirmed the presence of reciprocal translocation $\mathrm{t}(9 ; 12)(q 34.1 ; q 11.2)$.

Our results confirmed that FISH technique is a rapid, sensitive, quantitative technique which can be used for the evaluation of CM Lusing peripheral blood. FISH helps in the detection of minimal residual disease and disease recurrence with small percentage of abnormal cells. In our experience, this situation is usually associated with very high WBC count which can result in increase in the percentage of Phpositive cells.
\end{abstract}

Keywords: Fluorescence in situ hybridization (FISH), chromosomal analysis, chronic myeloid leukemia, peripheral blood.

\section{Introduction}

Chronic myeloid leukaemia (CML) is a clonal myeloproliferative disorder resulting from neoplastic transformation of haemopoietic stem cells that can lead to increase of myeloid series in peripheral blood and myeloid hyperplasia in bone marrow. The most common adult leukemia in India is CML with annual incidence ranging from 0.8-2.2/100,000 population in males and $0.6-1.6 / 100,000$ Access this article online Quick Response Code

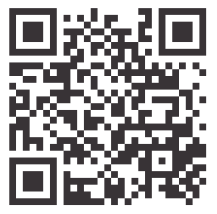

population in females [1].

The discovery of the first chromosomal abnormality in $C M L$ was done by Nowell and Hungerford in 1960 in Philadelphia and the deletion of one of the shortened chromosome of chromosomes 22 pair was named as 'Philadelphia chromosome' to honor the city [2]. The presence of Philadelphia chromosome (Ph) differentiates the chronic myeloid leukaemia from other chronic myeloproliferative disorders [3].

Philadelphia chromosome arising from the reciprocal translocation between long arm of chromosomes 9 and 22 that fuses the $A B L$ (Abelson Murine leukemia Virus) oncogene on chromosome 9 to the BCR (breakpoint cluster region) gene on chromosome 22 . This results in the formation of chimeric fusion gene BCR-ABL and this translocation - $t(9 ; 22)(q 34 ; q 11.2)$ was identified with advancement of banding techniques which became the diagnostic hallmark of CM L. Conventional cytogenetics or karyotyping is the standard method to detect 
chromosomal aberration. In addition to Ph chromosome, chromosomal abnormalities like trisomy 8 and isochromosome 17 also can be identified in CM L cases by using chromosomal analysis [3].

Fluorescence in situ hybridization (FISH) is a molecular cytogenetics technique, becoming a vital part of clinical practice in the workup of patients with hematological malignancies. FISH can be used to investigate both proliferating (metaphase cells) and non-proliferating (interphase nuclei) cells by detecting the location of specific nucleic acid sequences, using fluorescently labeled specific DNA probes to the target sample. FISH has been used as the most reliable method to identify the BCR/ABL gene at the time of diagnosis [3].

\section{Materialsand M ethods Subject}

A 52 year old female presented with fatigue and fever of one month duration was admitted in K.S. Hegde Charitable hospital, Deralakatte, M angalore. On examination, she had pallor and splenomegaly $4 \mathrm{cms}$ below the costal margin. The blood investigations revealed total WBC count of $2.5 \times 10^{9} / \mathrm{L}$ and hemoglobin level of $9 \mathrm{gms} / \mathrm{dl}$. Peripheral blood smear confirmed the diagnosis of CML. (Differential count -14 nRBCs/100 WBC, Myeloblast - 11\%, Promyelocytes - $7 \%$, M yelocytes - $21 \%$, M etamyelocytes $13 \%$, Band forms - $12 \%$, Neutrophils - $23 \%$, Eosinophils $8 \%$, Basophils - $5 \%$.) Peripheral blood smear picture of the patient is shown in Figure $1 \& 2$.

Owing to her high WBC count, $3 \mathrm{ml}$ of peripheral blood was collected in sodium heparin vacutainer tube and sent for karyotyping and Fluorescence in situ hybridization (FISH) to Diagnostic Centre for Cytogenetics and Molecular genetics.

\section{Conventional cytogenetic analysis}

Direct and 24 hour cultures were set up with peripheral blood using RPMI 1640 medium supplemented with 20\% of fetal bovine serum. GTG banding was done using standard cytogenetic protocol. M etaphases were analyzed from each culture, captured using the Olympus BX53 microscope and karyotyped with the 'GENASIS' software. $\mathrm{Ph}$ chromosome positivity was expressed in all 20 metaphases analyzed and the karyotype was reported according to An International System for Human Cytogenetic Nomenclature (ISCN) 2013.

\section{Fluorescence in situ hybridization (FISH) Analysis}

FISH analysis was carried out on interphase cells and metaphases of the fixed cell pellet using BCR/ABL Translocation, Dual fusion probe (Cytocell, UK). The $\mathrm{BCR} / \mathrm{ABL}$ probe set comprises a BCR probe mix directly labeled in green (Spectrum Green) spanning at 22q11.22q11-23 and the ABL1 probe directly labeled in red (Spectrum Orange) spanning at 9q34.11-q34.12. Cell pellet was dropped on the slide and $10 \mu \mathrm{l}$ of probe was applied to the target area. The sample and probe were co-denatured and hybridized using the ThermoBrite Denaturation / Hybridization System.

The ThermoBrite unit was programmed to allow 5 minutes of denaturation at $73^{\circ} \mathrm{C}$ to transform the double strands DNA into a single strand, followed by overnight hybridization at $37^{\circ} \mathrm{C}$ for the probe to hybridize with the target cells and bind to it. Next day, the slide was washed with $0.4 \mathrm{XSSC} / 0.1 \% \mathrm{NP}-40$ at $72^{\circ} \mathrm{C}$ for 2 minutes, followed by a wash in $2 \times \mathrm{SSC} / 0.1 \% \mathrm{NP}-40$ at room temperature for 1 minute to remove any of the probes that did not bind to the cells. The slide was air dried in the dark, then added $10 \mu$ l of DAPI (4, 6-diamidino-2-phenylindole) as a counter stain.

The FISH signals were visualized using an Olympus BX53 fluorescence microscope equipped with appropriate filters. Interphase nuclei and available metaphases were scored and the signals were captured using a CCD camera attached to a FISH View image acquisition and analysis system for FISH (GENASIS, Applied Spectral Imaging, Germany).

\section{Results}

Cytogenetic analysis of a metaphase showing $46, \mathrm{XX}, \mathrm{t}(9$; 22) (q34;q11.2) karyotype, which results in the Philadelphia chromosome is shown in Figure 3. FISH analysis of normal interphase nuclei will have a simple $2 \mathrm{R}, 2 \mathrm{G}$ signal pattern. 
As a result of the Philadelphia chromosome, ABL1 (Abelson) proto-oncogene and the BCR (Break point Cluster Region) gene fuse, giving rise to the BCR/ABL1 fusion gene showed 1 green, 1 red and 2 yellow fusion signals (1G, 1R, 2Y) in all the cells analysed (Figure $4 \& 5$ ).
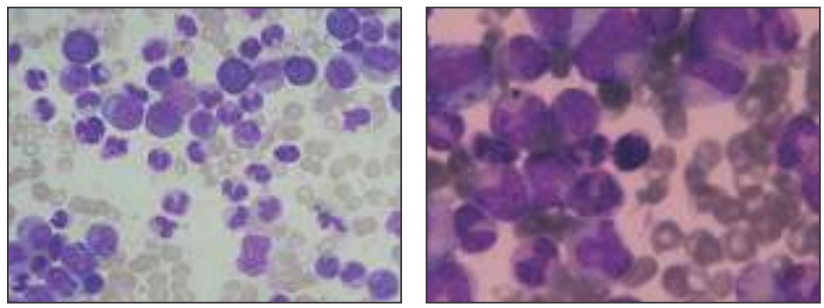

Figure1 \& 2 : Peripheral blood smear - Leishman's stain x400 and $x 1000$ Observe increased WBC count and the presence of increased myeloid series cells.

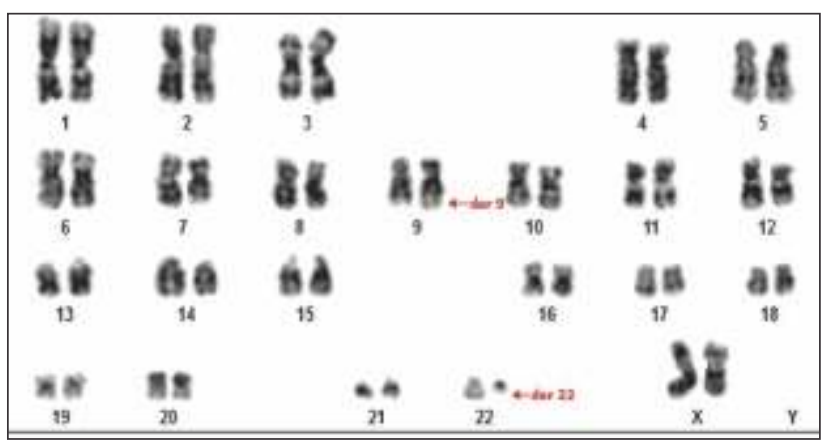

Figure 3 : GTG-banded karyogram of the patient showing $46, X X, t(9 ; 22)(q 34 ; q 11.2)$
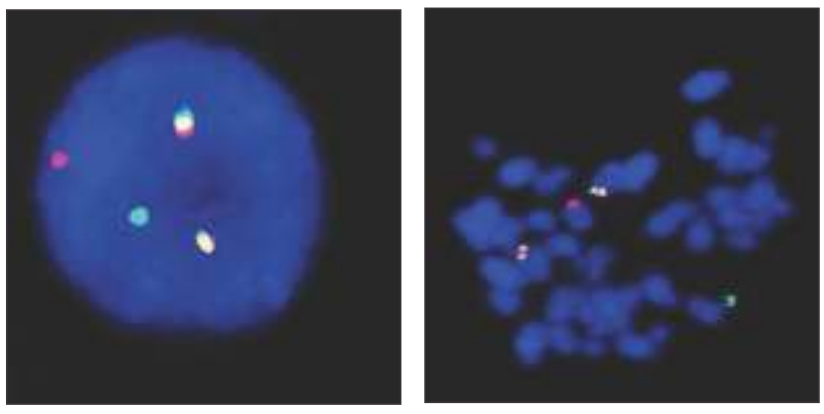

Figure $4 \& 5$ : Representative interphase cell and metaphase showing $B C R / A B L$ fusion as yellow signals, which represents overlapping of $B C R$ (green) and $A B L$ (red) signals. The other red signal represents $A B L$ on chromosome 9 and green represents BCRonchromosome 22.

\section{Discussion}

Ph chromosome is present in 95\% of CML cases and also present in $5 \%$ of children and $20 \%$ of adults with acute Iymphoblastic leukemia (ALL) [3]. Conventional cytogenetics is considered as the diagnostic hallmark for identification of $\mathrm{Ph}$ positive metaphases in $\mathrm{CML}$ cases when it is present in more than $30 \%$ cells. This method has its own limitations like increased turn around time, labour intensive procedure and analysis dependent on high numbers of viable dividing cells and well spread metaphases. Reena et al studied the sensitivity, specificity, positive predictive value (PPV) and negative predictive value (NPV) of conventional karyotyping, reported as $85 \%$, $100 \%, 100 \%$ and $71 \%$ respectively. The accuracy for conventional karyotyping method was $88 \%$ [3]. In the present study, conventional cytogenetics was done with 20 metaphase spreads and the Philadelphia chromosome $(\mathrm{Ph}+)$ was seen in all well spread metaphases. Good metaphase spreads are difficult to get and may consume extra time for analysis. However, conventional cytogenetics has its strength of detecting additional nonrandom chromosomal aberrations within the 20 spreads [4].

The quantification of BCR-ABL positive cells by FISH facilitates the monitoring of disease response to therapy [2]. The accuracy of FISH in the analysis of BCR-ABL rearrangements of $C M L$ patients has been demonstrated by several recent studies $[5,6,7]$. Even $1-2 \%$ of positive cells can be picked up due to the sensitivity of FISH test [5]. FISH was carried out in this patient with 200 interphase nuclei and available metaphases. The presence of $\mathrm{Ph}$ chromosome was confirmed and proved the accuracy of FISH on peripheral blood which was $100 \%$. This technique has many advantages, which include analysis of large number of cells, speed of reporting within 24 hours of the sample received in the laboratory, which may be useful in urgent diagnostic situations. It can be performed with fixed cell pellets left over from cytogenetic study of bone marrow or peripheral blood and paraffin embedded tissues[3].

Le Gouill et al studied the correlation between FISH and cytogenetics on bone marrow and got highly correlated results, then they moved on to the analysis of peripheral blood samples where comparable results were obtained, with an excellent correlation $(r=.97)$ [6]. Earlier reports 
have demonstrated that FISH technique was able to detect all types of BCR-ABL fusions in CM L with masked or variant $\mathrm{Ph}$, which is not apparent with the conventional karyotyping $[6,8]$. Lim et al observed additional karyotypic changes like supernumerary Ph or deletions of $9 q$ and/or $22 \mathrm{q}$ in $28.1 \%$ of Ph positive patients by interphase FISH [8]. The detection of BCR-ABL cells by Interphase FISH correlates better with molecular response, the exact interpretation of FISH in conjunction with chromosomal analysis is the best practice, to avoid the possibility of misdiagnosis $[8,9]$.

Patel et al observed significantly reduced disease free survival in patients with deletion in $\mathrm{ABL}, \mathrm{BCR}$ or $\mathrm{ABL}, \mathrm{BCR}$ on derivative 9 as compared to non-deleted derivative 9 and their findings suggest that the gene segments around the breakpoint are deleted in heterogeneous patterns when the translocation occurs and the genes located near the breakpoint may also play a role in the delayed tumor progression [10]. Thus, FISH technique gives information about the phases of CM L and useful at diagnosis after BMT

\section{References}

1. Subramaniyan PG. Cytogenetic study in CML. Indian J Med Res. 2012;135:12-13

2. Jha $C B$, Kucheria K, Choudhary VP. Diagnostic role of conventional cytogenetics and fluorescence in situ hybridization (fish) in chronic myeloid leukemia patients. Kathmandu University Medical Journal. 2006;4(2):171-175.

3. Reena RM Z, Julia M unchar MJ, Salwati S, Zubaidah Z, Hamidah NH, Sharifah NA, Cheong SK. Detection of BCR/ABL Gene in Chronic M yeloid Leukaemia: Comparison of Fluorescence in situ Hybridisation (FISH), Conventional Cytogenetics and Polymerase Chain Reaction (PCR) Techniques. M ed \& Health. 2006;1(1):5-13.

4. Anand MS, Varma N, Varma S, Rana KS, Malhotra P. Cytogenetic \& molecular analyses in adult chronic myelogenous leukaemia patients in north India. IJM R. 2012;135:42-48

5. Madon P, Athalye A, Bandkar V, Dhumal S, Sopariwala A, Parikh F. Fluorescence in-situ Hybridization (FISH) - A Rapid and Useful Technique for Diagnosis and Management in Leukemia. Int J Hum Genet. 2003;3(2):115-119.

6. Le Gouill S, Talmant P, Milpied N, Daviet A, Ancelot M, Moreau P, Harousseau JL, Bataille R, Avet-Loiseau H. Fluorescence In Situ Hybridization on Peripheral-Blood Specimens Is a Reliable M ethod to
(Bone Marrow transplantation) for detection of residual diseases.

\section{Conclusion}

Chromosomal analysis and FISH both have their advantages and should ideally be carried out together in order to make a correct diagnosis and predict/monitor response to newer molecular targeted treatment modalities like imatinib mesylate. FISH can be done in peripheral blood when the WBC count is high which can result in increase in percentage of $\mathrm{Ph}$ positive cells and also failed cases of conventional cytogenetic analysis to quantify disease in CML. FISH on peripheral blood exhibited a high degree of correlation with bone marrow, as a rapid, accurate and relatively non-invasive method.

\section{Acknowledgement}

The authors wish to thank the patient for providing the sample and written informed consent form (ICF). The authors are also grateful to Nitte University Ethics Committee for publication approval and to M rs. Vani Shetty forher technical help.

Evaluate Cytogenetic Response in Chronic Myeloid Leukemia. Journal of Clinical Oncology. 2000;18(7):1533-1538.

7. Landstrom AP, Ketterling RP, Knudson RA, Tefferi A. Utility of peripheral blood dual color, double fusion fluorescent in situ hybridization for $\mathrm{BCR} / \mathrm{ABL}$ fusion to assess cytogenetic remission status in chronic myeloid leukemia. Leukemia \& Lymphoma. 2006;47(10):2055-2061. DOI: $10.1080 / 10428190600783551$

8. Lim TH, Tien SL, Lim P, Lim AST. The Incidence and Patterns of BCR/ABL Rearrangements in Chronic Myeloid Leukaemia (CML) Using Fluorescence in-situ Hybridization (FISH). Ann Acad Med Singapore. 2005;34:533-538.

9. Testoni N, M arzocchi G, Luatti S, Amabile M , Baldazzi C, Stacchini M, et al,. Chronic myeloid leukemia: a prospective comparison of interphase fluorescence in situ hybridization and chromosome banding analysis for the definition of complete cytogenetic response: a study of the GIM EM ACM LW P. BLOOD. 2009;14(24):4939-4943. doi:10.1182/blood-2009-07-229864.

10. Patel BP, Trivedi PJ, Brahmbhatt MM, Gajjar SB, lyer RR, Dalal EN, Shukla SN, Shah PM. Deletion of derivative ABL, BCR or ABL-BCR fusion gene is associated with shorter disease free survival in CML patients. Int. J. Genet. Mol. Biol. 2010;2(7):158-164. 